\title{
The Current Situation, Analysis and Exploration on the Needs of Data Sharing among Government Departments: Based on the Empirical Study of Y city
}

\author{
Li Chongzhao \\ Yunnan University of Finance and Economics \\ Public Management School \\ Kunming, Yunnan, P.R. China, 650221 \\ (77812032@qq.com)
}

\author{
Qu Haiping \\ Yuxi Normal University \\ Foreign Language School \\ Yuxi, Yunnan, P.R. China, 653100 \\ (echoqu@126.com)
}

\begin{abstract}
In the era of big data, it is a key standard to measure the capacity of government management as to whether a government can manage and use data effectively and improve the level of data management and services. The paper takes 66 municipal government departments as study object, analyzes the key problems and factors hindering and restraining data sharing among government departments from the aspects of the present situation of data acquiring, storing, using and sharing, as well as the needs of sharing data in cooperative work. Finally the paper puts forward some solutions and suggestions to the problems.
\end{abstract} needs

Keywords-government departments; data resources; sharing

\section{INTRODUCTION}

The mingling of information technology and economic society brings the quick growth of data, and data has become essential strategic resources for each nation, which has increasingly had an enormous impact on the activity of global production, circulation, distribution and consumption, even economic operating mechanism, social lifestyles and nation governance capacities. Since 1990s, "18 Golds" led by Chinese government has effectively pushed forward the information construction of governmental core business, and enhanced data collection and transformation in vertical departments. However, influenced by government administrative system of binary matrix organization structure and barriers among different government departments, horizontal function departments perform their own functions separately without constraints, so the barriers lead to highly scattered and disordered data, shaping "Isolated Information Islands" and "Information Silo". Therefore, if government, as the biggest owner of data resources, can gradually realize how to manage and use the data resources fully, the administrative effectiveness and service level of governments will be greatly improved.

To put forward data sharing across different departments and levels, it's necessary to investigate the current situation of data collecting, storing, using and sharing, to know the needs of data sharing to optimize cooperative business among different departments, to find out the core departments and key fields requiring data sharing, to analyze the main problems impeding data association and sharing among government departments, and to explore the targeted and operative approach to data association and sharing.

\section{InVEStigAtion Method}

To reflect the current needs and situation of data sharing among government departments, after contrasting and referring to the methods of similar investigations, the team adopted questionnaire and depth interview, together with collecting, analyzing and coding second-hand information plus large sampling to carry out the investigation.

\section{A. Questionnaire}

Questionnaire design. After collecting and sorting the information of Chinese local governments conducting survey on the current situation and needs of data sharing, the team adopted Delphi method to design the questionnaire of data resources survey and the needs of data sharing in government departments. There was a small adjusting survey in the departments before the team revised ambiguity problems and incomplete options and finally finalized the questionnaire. "The Diagnostic Questionnaire on Data Resources of Government" mainly includes the name and description of datasets, the ranges of data, the existing states of data, secret or non-secret, data origin, and whether data is shared with other government departments and so on. "The Questionnaire on the Needs of Data Sharing among Government Departments" mainly includes 6 items, they are the name of datasets in need of sharing, the description of datasets, the requirement specification of shared data, the existing states of expected data, government units where data is probably offered, and expected period of data update. 
Questionnaire Distribution and Recovery. On July21, 2015, approved by General Office of Y City Municipal Government, the investigation team released "The Diagnostic Questionnaire on Data Resources of Government" and "The Questionnaire on the Needs of Data Sharing among Government Departments" to 66 municipal government departments through government Office Automation System. According to questionnaire instructions, each department and its branches filled in the questionnaire seriously. After our liaisons carefully examined the questionnaires to make sure there was no mistake or incomplete questions, all questionnaires were sent back to the investigation team mailbox before August $5,2015$.

\section{B. Depth Interview}

Design Interview Outline Interview outline had two steps. First, according to the result of questionnaire, the investigation team designed a draft of departmental interview outline based on open-ended questions to know data sharing demand and current situation in the process of optimizing department business. Second, we carried out a small adjusting research in municipal Bureau of Agriculture, so that we could revise and supplement a few unclear and ambiguous statements to get the final version of "The Interview Outline on the Needs of Data Sharing among Y City Government Departments".

Select Interview Departments. On the basis of data resources survey and investigation of data sharing needs, the team respectively sorted out the first 10 departments demanding for data most strongly, offering the most required data, and owning the most data. Deleting repeated departments, the investigation team contacted with left departments and finally interviewed 12 of them on site.

Collect Interview Data. Interview included group interview and individual interview. The team sent the interview outline to every respondent at first. As soon as respondents finished the outline, permitted by his department and himself, we began the open interview with recording and shorthand on site. Combined finished interview with shorthand draft, we converted recording into shorthand text and eventually we got 12 transcripts of departments interview.

\section{FINDINGS}

\section{A. The Statistics on Data Resources of Government Departments}

59 departments, totally 66 , took part in the investigation, accounting for $89.39 \%$ of the total. The 59 departments offered 1296 data items in 257 categories and 21.96 items per department on average. In 257 categories data, 165 categories $(49.5 \%)$ were stored in electronic documents, 79 categories $(23.70 \%)$ were stored in database, and 89 categories $(26.79 \%)$ were stored in paper. Within the 257 categories, 219 categories $(85.21 \%)$ were collected by departments themselves, and 38 categories $(14.79 \%)$ were acquired by sharing among departments.

\section{B. The Statistics on the Needs of Data Sharing among Departments}

38 departments, from totally 66 departments $(57.58 \%)$ involved in the research, asked 110 departments and units for 377 categories data, 2902 items in total. According to the belongings situation of data sharing needs, the track of it among departments was clear. $89.9 \%$ data was required from government, and $10.10 \%$ data was required from enterprises. According to data usage, it could be divided into elementary business data, the data of administrative approval, the data of administrative enforcement, planning data and statistical data. Each department demanded for elementary data most. Among 377 categories datasets required sharing, 374 categories described the data sharing situation, 328 categories were required sharing in electronic documents, 22 categories were hoped to be shared in paper document, and 24 categories were shared by using database. The update cycles of 377 categories database required sharing differed respectively. 193 items needs updating by the month, 119 items by the year, 21 items in real time, 14 items by the quarter, 13 items by the week, 11 items by the day, and 3 items by the developing cycle of projects.

\section{Problems}

Analyzing the data, we found out some problems of data sharing among government departments in Y city.

The collection of data is repeated, inefficient and in poor quality. According to the investigation, we found that the same data was collected by different departments repeatedly. When conducting their business, nearly 12 departments collected data from legal entities. Since the present system is incompatible or it's not supported with business system, most datasets were filled in and submitted in paper, and lots of data resources weren't stored electronically. It didn't only occupy spaces, but also need managing by exclusive person, which led to the waste of personnel and property. Therefore, the size of data and its storage way couldn't help departments to optimize business by using data analysis.

Undeveloped construction of information system in government operational departments and serious data fragmentation. The current business information system in municipal departments was constructed vertically under the lead of China central departments or provincial departments, while the municipal departments built a few on the need of their business. Due to lack of information-sharing platform, the present horizontal departments hardly shared information. Even different sectors didn't share data within the same department, and they still exchange data through paper or electronic documents.

Lack of long-acting standard of data sharing and security mechanism. 58.5\% departments involved into this survey asked for sharing data. On the one hand, because of lack of laws and policy documents permitting sharing data, some departments willing to exchange data didn't know the range, border or ways, so that their own data others required stuck inside, while the data they needed stayed outside. On the other hand, nearly $50 \%$ business data of government 
departments was stored in electronic document, so most documents were delivered through e-mail. When sharing bulk data in the process of collaborating business, they often copied the data with U-disk with the help of acquaintances or superiors. So it was clear that there was lack of security mechanism when sharing data safely.

Lack of awareness and motives of sharing data across different departments. A portion of staff taking part in the survey were not aware of the importance and necessity of data sharing, as well as the approaches and ways of it. They even thought sharing data was the process of delivering paper documents or electronic documents to some department or uploading a policy document on the website. Some departments, influenced by narrow-mindedness, took data resources as their own property, so that they were unwilling to share data.

\section{Analysis and advice}

After investigating and analyzing, we refined some practical approaches and ways to improve data sharing among government departments. First, it's crucial to strengthen top design and integrated planning, to draw up the catalogues and administrative measures of data resources, to define the boundaries and operation method, and to clarify the rights and compulsories in the process of data sharing and managing in each department. Second, it's important to establish the institution managing data professionally, to take charge of controlling and making the definition of data, to build up basic standard and regulation on managing data, to ensure the consistency and quality of data definition, to guide the process of developing information system, and to form the administrative notion of data-driven management and data-driven decision. Third, it's necessary to build up long-term mechanism of data collecting and sharing based on data resources catalogue, to make unified and standardized information collection table covering data collection needs of different levels, to eliminate different data acquisition channels, and to develop the mechanism, one data collection for multiple sharing. It's essential to manage and use data comprehensively, to realize mutual connection, communication, verification and correction of information, and to overcome inevitable data distortion in the process of manual data transmission. It's elementary to set up data sharing mechanism with rights and obligations being equivalent, to create data sharing surroundings, to improve the awareness of sharing data, to found a benefit-sharing pattern with data co-building and co-maintenance through multi-sectors action, and to improve the efficiency and quality of data collecting. Fourth, it's significant to enhance the capability of internet information security management and to effectively guarantee the security of public data closely bound up with the mass. Government should implement national classification protection system for information security, strengthen the management of internet and information security, carry out liability mechanism, improve the standard system of internet and information security, intensify internet management by law and protect personal information, make efforts to secure key information system and information infrastructure to ensure its safety and controllability.

\section{REFERENCES}

[1] Circular of the State Council on Printing and Issuing the Compendium of Promoting Big Data Action, promulgated by the State Council, January 2015.

[2] Mo Like, Wang Peimin, Transforming public information resources into a strategic national asset, Studies in Science of Science ,June 2004.

[3] Fan Bo, The Propelling System, Mechanism and Method of Information Sharing among Different Public Sectors, Journal of Shanghai Jiaotong University,January 2008.

[4] Zhang Nan, Sun Tao, Tang Jinhui, Public Data Management Based on Office Automation System, Chinese Public Administration,2008 Official Business Innovation Edition.

[5] Zhang Nan, Analyzing Public Generated Big Data and Restructuring Government Decision Making Process: Review and Prospect, Chinese Public Administration, October 2015.

[6] Li Weidong, The Theory and Method of Governmental Information Resources Sharing, Chinese Public Administration, January 2008.

[7] G. Eason, B. Noble, and I.N. Sneddon, "On certain integrals of Lipschitz-Hankel type involving products of Bessel functions," Phil. Trans. Roy. Soc. London, vol. A247, pp. 529-551, April 1955. (references) 